\title{
Le verbe terminologique : un portrait de travaux récents
}

\author{
L’Homme, Marie-Claude \\ Observatoire de linguistique Sens-Texte (OLST), Université de Montréal \\ mc.lhomme@umontreal.ca
}

\section{Introduction}

La terminologie s'étant longtemps concentrée sur la description des concepts (envisagés comme des représentations d'objets de connaissances) et sur la mise au jour des relations qu'ils partagent (genre espèce ; partie - tout), la discipline s'est surtout attardée sur la fonction dénominative des termes et sur leurs caractéristiques idéales de ce point de vue. Cette observation a de quoi surprendre en linguistique où le verbe occupe une place centrale dans presque tous les cadres théoriques. La perspective conceptuelle longtemps adoptée par la terminologie l'a amenée à se focaliser sur les unités de nature nominale et à faire l'impasse sur le fonctionnement linguistique ou textuel des termes. Les modèles terminologiques fondateurs (d'obédience conceptuelle) se prêtent mal à la prise en compte d'unités lexicales de nature prédicative (les verbes, notamment).

Toutefois, depuis près d'une vingtaine d'années, de nouveaux paradigmes remettent en question cette attention exclusive accordée au nom et proposent d'inclure les termes appartenant à d'autres parties du discours dans les ressources terminologiques. Les modèles descriptifs proposés s'inspirent de différents courants de la sémantique lexicale et de la linguistique de corpus.

Dans le présent article, nous nous attarderons sur les travaux ayant cherché à caractériser la nature spécialisée du verbe ou, encore, à mieux comprendre son aptitude à exprimer des sens spécialisés (des sens liés à des domaines de spécialité, comme le droit, l'informatique ou la médecine). Nous examinerons la question selon différentes perspectives : d'un point de vue conceptuel (section 2), à partir de ce que perçoivent les locuteurs lorsqu'on leur demande si un verbe est spécialisé (section 3), à partir de données issues de corpus spécialisés (section 4) et, enfin, depuis quelques travaux issus de travaux de sémantique lexicale (section 5). Nous examinerons également à la section 6 quelques modèles de description lexicographique ou terminographique des verbes. Nous conclurons en résumant les idées maîtresses qui se dégagent de ces travaux.

\section{Verbe et perspective conceptuelle}

Bien que le verbe ne soit pas complètement absent de la perspective conceptuelle (associée à la théorie fondatrice de la terminologie), il semble que son intérêt réside dans son aptitude à désigner des concepts d'activités (également exprimés par des noms). «Le verbe n'est terminologique que dans la mesure où son contenu conceptuel est assimilable à celui d'un nom» résume assez fidèlement les observations trouvées dans la littérature. Rey et Sager ont décrit la place du verbe en terminologie « conceptuelle » des manières suivantes :

La terminologie ne s'intéresse aux signes (mots et unités plus grandes que le mot) qu'en tant qu'ils fonctionnent comme des noms dénotant des objets et comme des « indicateurs de notions » (de concepts). Dans cette optique, les verbes sont des noms de processus, d'action (Rey $1979: 25)$.

Concepts represented in terminological dictionaries are predominantly expressed in the form of nouns; concepts which are linguistically expressed as adjectives and verbs in 
technical languages are frequently found only in the corresponding noun form and some

theorists deny the existence of adjective and verb concepts (Sager $1990: 58$ ).

Ce point de vue explique en partie le déséquilibre existant entre les parties du discours décrites dans un dictionnaire terminologique. La nomenclature d'un dictionnaire spécialisé comporte une très grande proportion de termes de nature nominale (des termes simples - biotechnologie, virus, bus - et des termes complexes - traitement de texte, réchauffement climatique); les verbes et les adjectifs intégrés à la nomenclature font figure d'exceptions.

Ainsi, une démarche résolument conceptuelle (incarnée aujourd'hui par les modélisations ontologiques) dégagera des concepts d'activité dans un domaine de spécialité (une classe de concepts qui prendra place à côté de celle des entités) et déclarera que ces concepts peuvent être exprimés par des noms ou des verbes. Ainsi, le verbe traiter devient terminologique dans la mesure où il constitue une variante linguistique servant à étiqueter "traitement de données "; la même remarque s'applique au verbe développer qui devient terminologique dans la mesure où il désigne le concept de « développement ».

La perspective conceptuelle peut également s'intéresser au verbe lorsqu'il exprime une caractéristique (par exemple, l'utilisation typique d'une entité) :

Souris : utilisée pour cliquer, glisser-déposer, etc.

Internet : réseau dans lequel un utilisateur peut $\underline{\text { naviguer }}{ }^{1}$

\section{Verbe et intuitions de locuteurs}

On peut se demander si le point de vue conceptuel tel que nous l'avons décrit dans la section précédente est en phase avec les intuitions que peuvent avoir des locuteurs sur le statut spécialisé ou terminologique de certaines formes verbales. C'est ce que nous avons tenté de caractériser au moyen d'un petit questionnaire décrit à la sous-section suivante. Nous verrons également que certains auteurs proposent des explications possibles de ce qui est perçu par les locuteurs.

\subsection{Une entrée en matière}

Nous avons distribué un questionnaire dans l'un de nos cours portant sur la langue de spécialité (voir Tableau 1). Nous avons demandé aux étudiants (tous inscrits à un programme de maîtrise en traduction) de dire s'ils considéraient que les verbes apparaissant en caractères gras dans les phrases étaient spécialisés. Ils devaient ensuite dire s'ils étaient certains de la réponse qu'ils donnaient ou s'ils hésitaient à l'endosser complètement.

Parmi les quinze verbes apparaissant dans les phrases, trois ou quatre sont considérés par tous comme étant spécialisés (naviguer, coder, synthétiser; télécharger - un seul répondant considère que ce dernier verbe n'est pas spécialisé). Cinq verbes sont définis par une majorité de répondants (cinq ou six) comme étant spécialisés (activer, écrire 2 , programmer, cliquer). Deux verbes ne semblent pas avoir de sens spécialisé pour une majorité de répondants (développer, fondre). Les autres verbes ont été visiblement plus difficiles à classer.

Tableau 1 : Perception de huit répondants quant à la nature spécialisée de certains verbes 


\begin{tabular}{|c|c|c|}
\hline Phrase & $\begin{array}{c}\text { Spécialisé ? (oui ou } \\
\text { non) }\end{array}$ & $\begin{array}{l}\text { Certain? (ou } \\
\text { non) }\end{array}$ \\
\hline \multirow[t]{3}{*}{$\begin{array}{l}\text { [Environnement] C'est pour cela que l'on dit que la } \\
\text { planète se réchauffe. }\end{array}$} & 4 oui & $\begin{array}{l}2 \text { certains / } 2 \\
\text { non certains }\end{array}$ \\
\hline & 3 non & 3 non certains \\
\hline & 1 sans réponse & 1 sans réponse \\
\hline \multirow[t]{2}{*}{$\begin{array}{l}\text { [Environnement] L'extraction ou la récolte de ces } \\
\text { ressources peuvent également polluer le sol, l'eau et } \\
\text { l'air. }\end{array}$} & 4 oui & $\begin{array}{l}1 \text { certain / } 2 \text { non } \\
\text { certains / } 1 \text { sans } \\
\text { réponse }\end{array}$ \\
\hline & 4 non & $\begin{array}{l}3 \text { certains / } 1 \\
\text { non certain }\end{array}$ \\
\hline \multirow{2}{*}{$\begin{array}{l}\text { [Environnement] En outre, les glaciers de montagne } \\
\text { du monde entier devraient continuer à fondre, ce qui } \\
\text { ajouterait de } 5 \text { à } 15 \mathrm{~cm} \text { d'eau supplémentaire, leur } \\
\text { eau se retrouvant dans la mer. }\end{array}$} & 3 oui & 3 non certains \\
\hline & 5 non & $\begin{array}{l}4 \text { certains / } 1 \\
\text { non certain }\end{array}$ \\
\hline \multirow{2}{*}{$\begin{array}{l}\text { [Informatique] Le programmeur écrit son } \\
\text { programme dans un langage de programmation à } \\
\text { l'aide d'un éditeur. }\end{array}$} & 4 oui & 4 certains \\
\hline & 4 non & $\begin{array}{l}3 \text { certains / } 1 \\
\text { non certain }\end{array}$ \\
\hline \multirow[b]{2}{*}{$\begin{array}{l}\text { [Informatique] Bien que ces unités portent le nom de } \\
\text { «lecteur», elles servent néanmoins a la fois a lire et à } \\
\text { écrire des données. }\end{array}$} & 5 oui & 5 certains \\
\hline & 3 non & $\begin{array}{l}1 \text { certain / } 2 \text { non } \\
\text { certains }\end{array}$ \\
\hline \multirow{2}{*}{$\begin{array}{l}\text { [Informatique] La nouvelle version peut être } \\
\text { téléchargée à partir de cette adresse. }\end{array}$} & 7 oui & 7 certains \\
\hline & 1 non & 1 certain \\
\hline $\begin{array}{l}\text { [Informatique] Avec ce type de connexion, vous } \\
\text { pouvez naviguer rapidement dans Internet. }\end{array}$ & 8 oui & $\begin{array}{l}7 \text { certains / } 1 \\
\text { non certain }\end{array}$ \\
\hline \multirow{2}{*}{$\begin{array}{l}\text { [Informatique] ... cliquer sur le bouton fermer dans } \\
\text { le coin supérieur droit de la fenêtre du programme. }\end{array}$} & 6 oui & 6 certains \\
\hline & 2 non & 2 certains \\
\hline \multirow{2}{*}{$\begin{array}{l}\text { [Informatique] Ainsi, en } \mathrm{C}++ \text {, il est toujours } \\
\text { possible de programmer sans utiliser la bibliothèque } \\
\text { objet, ce qui est beaucoup moins naturel en Java. }\end{array}$} & 6 oui & $\begin{array}{l}5 \text { certains } / 1 \\
\text { non certain }\end{array}$ \\
\hline & 2 non & 2 certains \\
\hline \multirow[t]{2}{*}{$\begin{array}{l}\text { [Informatique] La suite Office a été développée par } \\
\text { la compagnie Microsoft. }\end{array}$} & 2 oui & $\begin{array}{l}1 \text { certain / } 1 \text { non } \\
\text { certain }\end{array}$ \\
\hline & 6 non & $\begin{array}{l}5 \text { certains / } 1 \\
\text { non certain }\end{array}$ \\
\hline \multirow{2}{*}{$\begin{array}{l}\text { [Informatique] Activer une fenêtre lorsqu'une fenêtre } \\
\text { est partiellement recouverte par une autre, un clic } \\
\text { suffit à l'activer. }\end{array}$} & 6 oui & $\begin{array}{l}5 \text { certains / } 1 \\
\text { non certain }\end{array}$ \\
\hline & 2 non & 2 certains \\
\hline \multirow{2}{*}{$\begin{array}{l}\text { [Médecine] Les espèces [de bactéries] anaérobies } \\
\text { sont pathogènes quand elles se multiplient d'une } \\
\text { façon exagérée dans son site normal et y deviennent } \\
\text { dominantes mais aussi et surtout quand elles }\end{array}$} & 4 oui & $\begin{array}{c}3 \text { certains / } 1 \\
\text { non certain }\end{array}$ \\
\hline & 4 non & 4 certains \\
\hline
\end{tabular}




\begin{tabular}{|l|c|c|}
\hline $\begin{array}{l}\text { colonisent un organe ou une cavité normalement } \\
\text { stérile (plèvre, poumons, péritoine, cerveau...). }\end{array}$ & & \\
\hline $\begin{array}{l}\text { [Médecine] La sécrétion d'entérotoxine est codée par } \\
\text { un plasmide. }\end{array}$ & 8 oui & 8 certains \\
\hline $\begin{array}{l}\text { [Médecine] Enfin, la symptomatologie évolue vers } \\
\text { un choc septique et le décès. }\end{array}$ & 4 oui & $\begin{array}{c}3 \text { certains } / 1 \\
\text { non certain }\end{array}$ \\
\cline { 2 - 3 } & 4 non & $\begin{array}{c}3 \text { certains } / 1 \\
\text { non certain }\end{array}$ \\
\hline $\begin{array}{l}\text { [Médecine] Les bactéries peuvent ensuite se se } \\
\text { développer et synthétiser la toxine. }\end{array}$ & $\mathbf{8}$ oui & 8 certains \\
\hline
\end{tabular}

Indépendamment des écarts entre les réponses, ce qui ressort nettement de ce petit questionnaire est le fait que la totalité des répondants considèrent que certains verbes peuvent véhiculer un sens spécialisé. Il est toutefois difficile - à la lumière des données dont nous disposons - d'identifier clairement les raisons pour lesquelles certains verbes sont spécialisés pour une majorité de répondants et d'autres non. Leur intuition peut être guidée par des considérations comme les suivantes :

- Emplois divergeant d'emplois connus : naviguer, coder;

- Verbes n'existant que dans un domaine spécialisé : synthétiser, télécharger, cliquer;

- Activités fortement associées à un domaine : activer (une fenêtre), programmer, écrire 2 (un programme).

Par ailleurs, on remarque que les répondants ont éprouvé plus de difficulté à statuer sur la nature spécialisée des verbes dont le sens ne s'éloigne pas suffisamment de sens familiers (réchauffer, polluer, coloniser, écrire ${ }_{1}$ ). Les intuitions de nos répondants semblent guidées par des considérations autres que conceptuelles (c'est-à-dire sur l'aptitude d'un verbe à exprimer un concept ou la caractéristique d'un concept). Par exemple, les verbes télécharger et cliquer font sans doute l'unanimité en raison du fait qu'ils sont exclusivement associés à des activités informatiques et non parce qu'ils servent de supports linguistiques à des concepts. Une autre observation se dégageant de l'enquête est que, pour les répondants, certains verbes sont plus spécialisés que d'autres.

\section{2 «Classes » de verbes spécialisés ?}

Des auteurs ont proposé de classer les verbes selon différents « niveaux de spécialisation ». Ces classements jettent un premier éclairage sur les données issues de notre petite enquête. Condamines (1993) propose une méthode permettant d'exploiter une caractérisation syntaxico-sémantique fine de verbes et de noms afin d'identifier des mots inconnus (noms et verbes). Appliquée au domaine bancaire, la méthode permet de découvrir : 1. des verbes spécifiques au domaine qui sont inconnus (ex. débiter); 2. des verbes connus mais véhiculant un sens inconnu dans le domaine (ex. retirer).

Utilisant une méthode qui s'appuie sur les classes d'objets (Gross 1994) auxquelles appartiennent les arguments des verbes, Lerat (2002) classe les verbes juridiques dans trois groupes distincts : 1. les verbes très spécialisés (ex. adjuger, abroger); 2. les verbes supports (ex. mettre dans mettre aux voix, porter dans porter atteinte); et 3. les verbes polysémiques dont au moins un sens est spécialisé (ex. 'HUMAIN contracter (v. intr.)' et 'HUMAIN contracter (v. tr.) $\mathrm{N}<$ obligation>' relèvent du domaine juridique alors que 'HUMAIN contracter (v. tr.) $\mathrm{N}<$ muscle $>$ ' relève du domaine médical). 
Enfin, dans un travail où l'auteure veut identifier les verbes participant à la transmission de connaissances spécialisées, Lorente (2002) propose un certain nombre de critères (voir section 5.3) pour l'élaboration d'une typologie des verbes, typologie selon laquelle les verbes se répartissent en quatre groupes distincts : 1 . verbes discursifs (permettant d'articuler le discours); 2. verbes connecteurs (exprimant des attributs, des équivalences, des relations de similarité ou de dépendance); 3. les verbes phraséologiques (se combinant avec un terme du domaine et exprimant avec ce terme, des connaissances spécialisées); 4. les verbes terminologiques (ayant un sens spécifique à l'intérieur d'un domaine spécialisé). Le dernier groupe renferme des verbes véhiculant des sens spécialisés; les deuxième et troisième groupes renferment des verbes qui peuvent véhiculer des connaissances spécialisées, mais n'ont pas à proprement parler un sens lié à un domaine de connaissance selon l'auteure. Le groupe des verbes discursifs ne renferme pas de verbes spécialisés selon l'auteure.

\section{Verbe et corpus}

Nous pouvons également tenter de déterminer dans quelle mesure les données issues des corpus spécialisés peuvent nous renseigner sur l'importance des verbes et, indirectement, sur leur aptitude à exprimer des sens spécialisés. Nous examinerons quelques données issues de corpus d'informatique et de changements climatiques en nous interrogeant sur les fréquences respectives des verbes et de leur nominalisation (Tableau 2).

On remarque que certaines nominalisations sont beaucoup plus fréquentes que le verbe correspondant. Toutefois, ce phénomène n'est pas systématique. Par exemple, dans les paires activer-activation et cliquerclic, c'est la forme verbale qui domine largement (rappelons également que activer et cliquer ont été considérés comme spécialisés par une majorité de répondants, voir section 3.1).

Tableau 2 : Fréquences respectives de verbes et de noms dans des corpus d'informatique et d'environnement

\begin{tabular}{|l|l|r|}
\hline Domaine & Forme lexicale $^{2}$ & Fréquence dans le corpus $^{-}$ \\
\hline Environnement & réchauffer & 192 \\
\hline & réchauffement & 1245 \\
\hline & polluer & 36 \\
\hline & pollution & 250 \\
\hline & fondre & 63 \\
\hline & fonte & 202 \\
\hline Informatique & naviguer & 114 \\
\hline & navigation & 204 \\
\hline & cliquer & 2358 \\
\hline & clic & 353 \\
\hline & développer & 376 \\
\hline & développement & 668 \\
\hline & activer & 870 \\
\hline & activation & 163 \\
\hline
\end{tabular}

L'interrogation des corpus amène également les terminologues à découvrir des formes lexicales formellement apparentées sur lesquelles il est difficile de faire l'impasse puisque cette similitude formelle 
s'accompagne souvent d'une parenté sémantique. Une interrogation à partir de la chaîne de caractères polludans un corpus d'environnement dévoilera la présence de formes lexicales comme polluant (adj.), polluant (n. m.), pollution (n. f.), pollueur (n. m.) et polluer (v. tr.), comme le montre la figure 1.

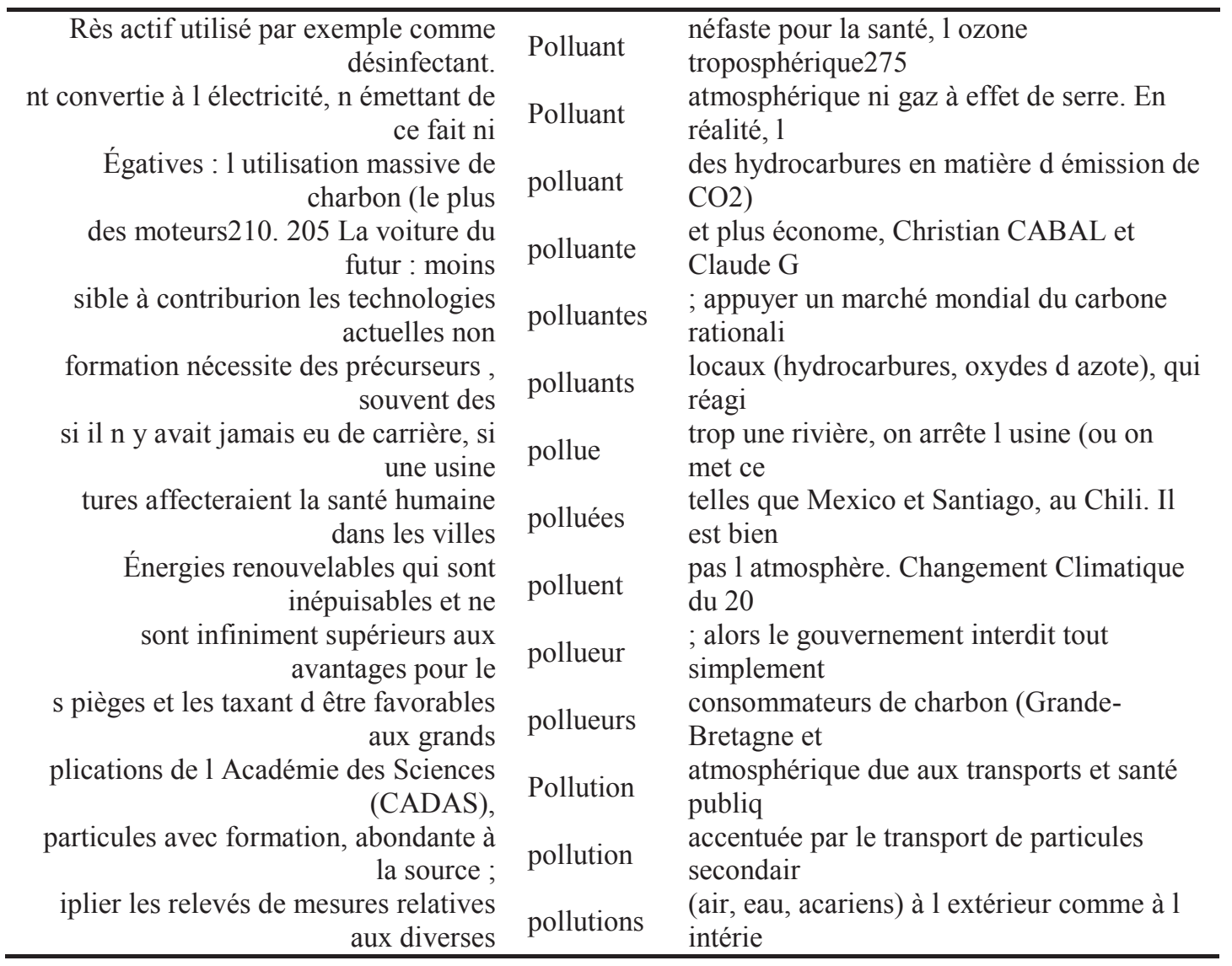

Figure 1 : Concordances obtenues à partir de la chaîne pollu- dans un corpus d'environnement

On remarque que les concordances de la figure 1 mettent au jour des séries lexicales qui engagent des termes appartenant aux parties du discours du nom, du verbe et de l'adjectif. L'examen attentif de ces concordances ou d'autres concordances semblables pourra mener à la découverte de réseaux lexico-terminologiques dans lesquels les unités sont reliées entre elles selon des modalités diverses que pourra caractériser et décrire la sémantique lexicale (voir les sections 5.2 et 6.2).

\section{Verbe et perspectives lexico-sémantiques}

La plupart des travaux ayant cherché à caractériser la nature spécialisée ou terminologique du verbe se situent dans un cadre théorique associé à la sémantique lexicale : classes d'objets (Gross 1994), Lexicologie explicative et combinatoire (Mel'čuk et al. 1995), Sémantique des cadres (Fillmore 1982). Comme on le verra dans les sous-sections suivantes, ces travaux ont mis au jour l'importance de tenir compte de la structure argumentale du verbe et son appartenance à un réseau lexical plus large. 


\subsection{Le verbe et sa structure argumentale}

Différents travaux ont montré que le verbe n'est pas spécialisé par lui-même. Étant une unité de nature prédicative (c'est-à-dire faisant appel à des participants appelés arguments ou actants selon le cadre utilisé pour les nommer), le verbe est spécialisé ou non dans la mesure où l'ensemble de sa structure argumentale est prise en compte.

Dans un travail (L'Homme 1998) où nous avons tenté de dégager des critères afin de statuer sur la nature terminologique des verbes, nous avons proposé un premier test qui consiste à tenir compte de la nature des arguments d'un verbe perçu intuitivement comme étant spécialisé. Ce critère permet d'admettre un verbe comme installer comme dans l'utilisateur installe la nouvelle version du traitement du texte sur son PC, car les réalisations des arguments du verbe installer - pris dans ce sens - sont réalisés au moyen de termes appartenant au domaine de l'informatique ${ }^{3}$.

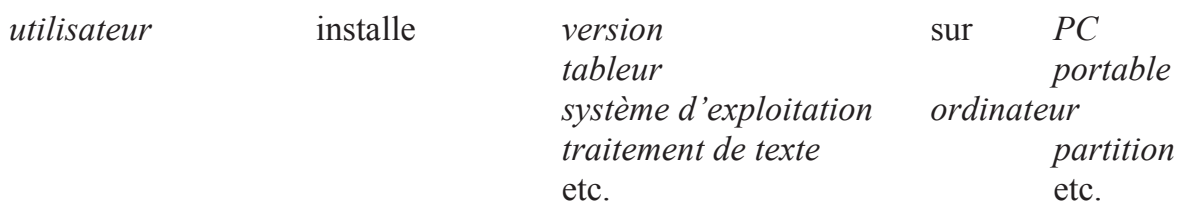

Ce test apparaît dans des formes similaires dans les travaux de Condamines, qui fait appel à une caractérisation des arguments des unités de nature prédicative, et dans ceux de Lerat (2002), qui dégage les classes d'objets auxquelles ils appartiennent. Il a également été repris comme un critère important dans les travaux de Pimentel (2012, à paraître) et Tellier (2007). La prise en compte de la nature des arguments permet de sortir de l'impasse (imposée d'une certaine manière par l'optique conceptuelle) que seuls les verbes associés étroitement à un nom peuvent prétendre au statut terminologique. Le critère présente également l'avantage de baliser l'intuition des locuteurs qui attribuent un sens spécialisé à des verbes dont le sens ne se distingue pas radicalement de sens connus (activer une fenêtre, écrire un programme); c'est sans doute en raison de la nature spécialisée plus facilement perceptible du second argument de ces verbes qu'ils sont perçus comme étant spécialisés.

\subsection{Appartenance du verbe à un réseau lexico-terminologique}

Outre la nature des actants, d'autres paramètres ont été évoqués par les auteurs pour statuer sur le caractère spécialisé des verbes. Un critère souvent évoqué est le lien qu'un verbe peut avoir avec un terme de nature nominale (Lorente 2002, 2007; Rey 1976; Sager 1990). Si le nom est terminologique et que le verbe est sémantiquement (et, souvent, morphologiquement) apparenté à celui-ci, il y a de fortes chances que le verbe soit spécialisé lui-même ${ }^{4}$.

Dans certains cas, le verbe et le nom expriment tous deux un sens d'activité (s'alignant ainsi sur les principes de l'optique conceptuelle) :

$\begin{array}{ll}\text { développement } & \text { développer } \\ \text { téléchargement } & \text { télécharger } \\ \text { réchauffement } & \text { réchauffer }\end{array}$

Dans d'autres cas, les sens des verbes et des noms sont reliés mais distincts : 
programme

programmeur pollution

polluant

pollueur programmer (programme est le résultat de l'activité de programmer)

programmer (programmeur est l'agent typique de programmer) polluer (pollution, dans l'une de ses acceptions, est le résultat de l'activité de polluer)

polluer (polluant est l'un des moyens typiques de polluer) polluer (pollueur est l'agent typique de polluer)

Dans cette perspective, souvent les noms ont servi de point de départ à partir duquel les verbes seront découverts en fonction des liens qu'ils partagent avec eux. Mais il n'est pas impossible de déplacer le point focal de l'analyse et d'y placer le verbe. Cela amènera le terminologue à découvrir d'autres unités plus directement reliées au verbe.

\begin{tabular}{|c|c|c|c|}
\hline Compilateur & $\begin{array}{l}\text { compiler } \\
\text { compilation }\end{array}$ & compiler & $\begin{array}{l}\text { compilateur } \\
\text { compilation } \\
\text { compilable } \\
\text { recompiler } \\
\text { recompilable } \\
\text { décompiler } \\
\text { etc. }\end{array}$ \\
\hline
\end{tabular}

Ce déplacement permet également de découvrir des liens engageant des verbes et d'autres unités lexicales qui ne sont pas apparentées morphologiquement. Nous avons suggéré (L’Homme 2003) que le verbe était particulièrement utile pour dévoiler ces réseaux sans doute davantage que les noms dénotant des entités. Comme le montre la figure 2, le verbe programmer au sens de « réaliser un programme en vue de réaliser une tâche », est relié à programmation et à programme, mais également à informaticien, à langage, à logiciel, à écrire 2 et à développer.

Figure 2 : Une partie du réseau lexical de programmer $_{1}$ (DicoInfo visuel, Robichaud 2011)

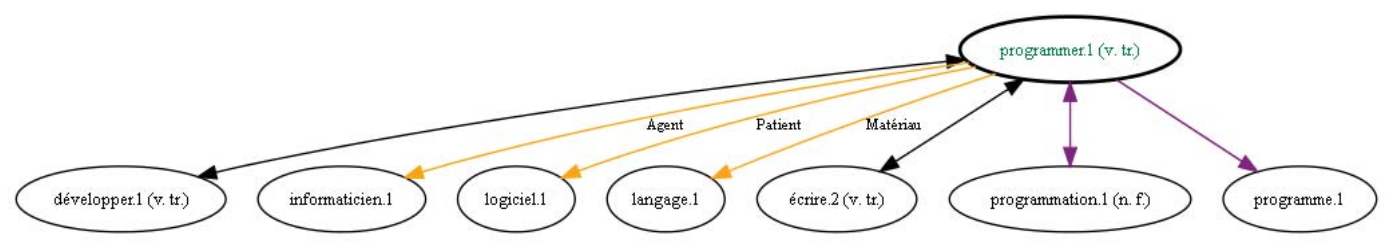

C'est la raison pour laquelle nous avons suggéré de combiner au critère de nature des arguments d'autres critères comme la prise en compte de liens morphologiques et paradigmatiques. 


\subsection{Une batterie de critères linguistiques}

La batterie de critères la plus extensive pour évaluer le caractère spécialisé des verbes est sans conteste celle de Lorente (2002) qui fait appel à différents paramètres syntaxiques, lexico-sémantiques et discursifs (sortant ainsi des sentiers balisés de la sémantique lexicale). Ces critères sont les suivants : la fonction discursive du verbe, sa valeur spécialisée, le contenu sémantique du groupe verbal, la formation morphologique des verbes, leur sous-catégorisation, la relation sémantique qu'ils partagent avec le sujet et la sélection lexicale dont ils sont le pivot. Lorente (2007) revisite cette longue liste en 2007 et propose de retenir deux paramètres seulement : le lien qu'on peut établir avec des termes de nature nominale et la classe sémantique des verbes. Dans cette deuxième version, la participation des verbes à la transmission de connaissance spécialisée passe par leur lien plus ou moins étroit avec des noms définis comme termes.

\section{Modèles descriptifs du verbe spécialisé}

Certains des travaux cités dans les pages qui précèdent ont abouti à la proposition de modèles descriptifs (lexicographiques ou terminographiques) conçus pour accueillir les caractéristiques propres aux verbes spécialisés. Les uns mettent l'accent sur une représentation de la structure argumentale mettant en évidence le statut spécialisé des verbes ; les autres, sur la mise au jour des réseaux lexicaux auxquels les verbes appartiennent.

\subsection{Structure argumentale}

La structure argumentale des verbes spécialisés a été décrite de différentes manières, mais toutes à mon avis sont compatibles. Comme nous l'avons vu plus haut, Lerat (2002) regroupe les réalisations concrètes d'arguments qui sont généralisées sous forme de classes d'objets. Tellier (2007) rend compte des arguments au moyen de classes sémantiques associées au domaine médical. Pimentel (2010) propose une caractérisation des arguments s'appuyant sur la Sémantique des cadres (Fillmore 1982) et formule, pour chacun des arguments de verbes juridiques anglais et portugais, des étiquettes pour les éléments de cadres (Frame elements). Dans deux dictionnaires spécialisés dont nous supervisons la compilation, le DiCoInfo ${ }^{5}$ et le DiCoEnviro ${ }^{6}$, nous avons proposé de rendre compte de la structure argumentale au moyen de deux systèmes: les rôles sémantiques et les termes typiques (L’Homme 2010). Le tableau 3 renferme des illustrations de ces quatre modèles.

Tableau 3 : Représentations diverses de la structure argumentale de verbes spécialisés

\begin{tabular}{|c|c|c|}
\hline Verbe & Structure argumentale & Auteur \\
\hline Contracter & Classes d'objets : HUMAIN contracter (v. tr.) $\mathrm{N}<$ obligation $>$ & $\begin{array}{l}\text { Lerat } \\
(2002)\end{array}$ \\
\hline contaminer & $\begin{array}{l}\text { Classes sémantiques : X (AGENT INFECTIEUX) Y (ANIMAL; } \\
\text { PRÉPARATION; SUBSTANCE BIOLOGIQUE; SUBSTANCE } \\
\text { COMPLEXE, MATÉRIAU) }\end{array}$ & $\begin{array}{l}\text { Tellier } \\
(2007)\end{array}$ \\
\hline acquit $_{1}$ & Étiquettes d'éléments d'un cadre : Judge $\sim$ Defendant of Charges & $\begin{array}{l}\text { Pimentel } \\
(2010)\end{array}$ \\
\hline télécharger & $\begin{array}{l}\text { Rôles sémantiques : Agent } \sim \text { Patient de Source à Destination } \\
\text { Termes typiques : Utilisateur } \sim \text { fichier; logiciel de réseau, ordinateur à } \\
\text { ordinateur }\end{array}$ & $\begin{array}{l}\text { L'Homme } \\
\text { (2010) }\end{array}$ \\
\hline
\end{tabular}


L’Homme (2009) et Pimentel (2012) proposent également de rendre compte des réalisations concrètes des arguments au moyen d'une annotation à la FrameNet (Rupenhoffer et al. 2010). Cette annotation permet de relier la structure argumentale (formulée par le lexicographe) et ses réalisations concrètes dans des phrases extraites de corpus. Comme le montre la figure 3, l'annotation met en évidence les fonctions et groupes syntaxiques des arguments (actant dans la figure) et les termes apparaissant dans telle ou telle position argumentale. D'autres groupes syntaxiques sont également annotés (autres, dans le tableau).

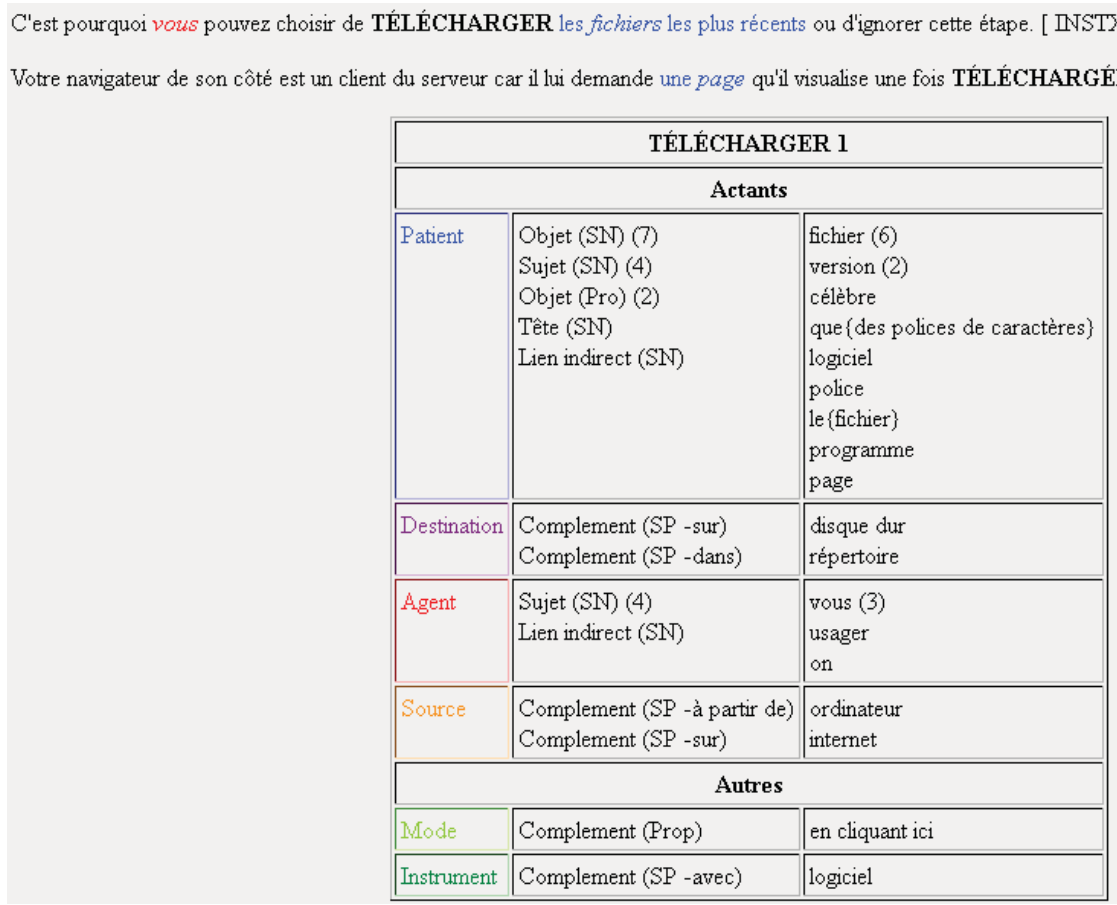

Figure 3 : Annotation de contextes

La prise en compte de la structure argumentale et les annotations de contextes ont été utilisées par Pimentel et al. (2011) afin de voir dans quelle mesure elles pouvaient servir à distinguer les sens propres à un domaine et les sens généraux de verbes anglais. En comparant les descriptions contenues dans le DiCoInfo et celles apparaissant dans la ressource lexicale FrameNet, les auteures ont identifié différents écarts plus ou moins prononcés avec les descriptions générales (le travail a été entrepris afin de voir si certains sens spécialisés pouvaient être ajoutés à la ressource lexicale) : 1. nombre différent d'arguments; 2 . nature différente des arguments; 3 . nombre et nature semblable des actants.

Cette comparaison permet de voir un certain nombre de différences entre les sens de execute décrits dans FrameNet (dans le cadre [Execution]) et dans le DiCoInfo (figure 4). La description de FrameNet montre que le premier argument est de nature animée alors que la plupart des réalisations d'arguments répertoriées dans le DiCoInfo dénotent des inanimés. 


\begin{tabular}{|c|c|c|}
\hline & $\begin{array}{l}\text { FrameNet } \\
\text { Execute [Execution] }\end{array}$ & $\begin{array}{l}\text { DiCoInfo } \\
\text { Execute } 2 b\end{array}$ \\
\hline $\begin{array}{l}\text { Core } \\
\text { FEs / } \\
\text { actantial } \\
\text { structure }\end{array}$ & Executioner EXECUTES Executed & Agent EXECUTES Patient \\
\hline \multirow{2}{*}{$\begin{array}{l}\text { Nature } \\
\text { of the } \\
\text { core FEs } \\
\text { / actants }\end{array}$} & $\begin{array}{l}\text { The Executioner is the individual who } \\
\text { performs the execution. } \\
\text { Semantic type: "Sentient" } \\
\text { Occurrences: government, army, state, } \\
\text { security forces, Saudi Arabia, etc. }\end{array}$ & $\begin{array}{l}\text { Agent: The participant that is } \\
\text { responsible for the action. } \\
\text { Occurrences: cpu, processor, you, we, } \\
\text { attacker, program, person, site, etc. }\end{array}$ \\
\hline & $\begin{array}{l}\text { The Executed is the person who is killed } \\
\text { by the Executioner in retribution for the } \\
\text { crime that the Executed was convicted of. } \\
\text { Semantic type: "Sentient" } \\
\text { Occurrences: prisoner, soldiers, people, } \\
\text { drug offenders, the King of France, etc. }\end{array}$ & $\begin{array}{l}\text { Patient: The participant that undergoes } \\
\text { the action. } \\
\text { Occurrences: command, instruction, } \\
\text { query, function, check, attack, macro, } \\
\text { etc. }\end{array}$ \\
\hline
\end{tabular}

Figure 4 : Descriptions du verbe execute dans FrameNet et dans le DiCiInfo (Pimentel et al. 2011).

L'examen de la structure argumentale des verbes peut également servir de paramètre de base pour établir des liens d'équivalence (ou, encore, observer des différences entre les réalisations des équivalents interlinguistiques). C'est ce qu'ont voulu vérifier Pimentel et L'Homme (2011) qui ont mené une étude comparative des structures argumentales et des annotations d'un certain nombre de verbes anglais et français extraits de la ressource spécialisée DiCoInfo. Ce travail a permis de donner un nouveau point de vue sur la relation d'équivalence en prenant en compte l'ensemble de la structure argumentale des verbes. Par exemple, les termes click et cliquer ont une structure argumentale faisant appel aux rôles sémantiques Agent, Patient et Instrument. Dans les contextes anglais annotés jusqu'ici, les arguments associés au rôle sémantique Instrument ne se réalisent jamais, tandis que dans les contextes français, ils sont instanciés dans trois cas (figure 5). 


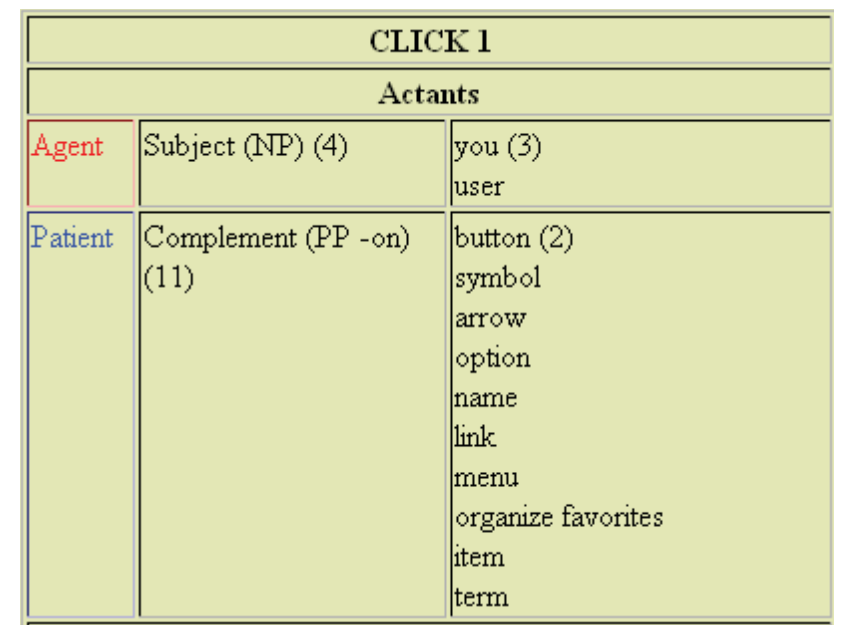

\begin{tabular}{|l|l|l|}
\hline \multicolumn{2}{|c|}{ CLIQUER 1 } \\
\hline \multicolumn{2}{|c|}{ Actants } \\
\hline Patient & $\begin{array}{l}\text { Complement } \\
\text { (SP -sur) (12) }\end{array}$ & $\begin{array}{l}\text { icône (3) } \\
\text { bouton (3) } \\
\text { ok } \\
\text { celle-ci } \\
\text { lien } \\
\text { suivant } \\
\text { onglet } \\
\text { démarrer }\end{array}$ \\
\hline Instrument & $\begin{array}{lll}\text { Complement } \\
\text { (SP -avec) (3) }\end{array}$ & $\begin{array}{l}\text { bouton (2) } \\
\text { souris }\end{array}$ \\
\hline Agent & $\begin{array}{lll}\text { Sujet (SN) (4) } \\
\text { vous (3) } \\
\text { il }\end{array}$ \\
\hline
\end{tabular}

Figure 5 : Tableaux associés aux annotations de click et cliquer (Pimentel e L'Homme 2011)

\subsection{Relations lexicales}

Certaines ressources spécialisées décrivent également une partie ou la totalité du réseau lexical dans lequel apparaissent les verbes. Tellier (2007) tient compte, par exemple de relations de synonymie, de quasisynonymie, d'antonymie et de différentes relations morphologiques (par exemple, le verbe sécréter est relié à sécrétion, sécréteur, sécrété, produire, excréter). Dans le DiCoInfo (L’Homme 2009), différentes relations lexicales sont dégagées entre les verbes retenus comme entrées et d'autres unités lexicales. Ces relations sont également décrites au moyen des fonctions lexicales de la lexicologie explicative et combinatoire (Mel'čuk et al. 1995). La figure 3 montre de quelle manière les relations lexicales sont décrites pour le verbe télécharger. 


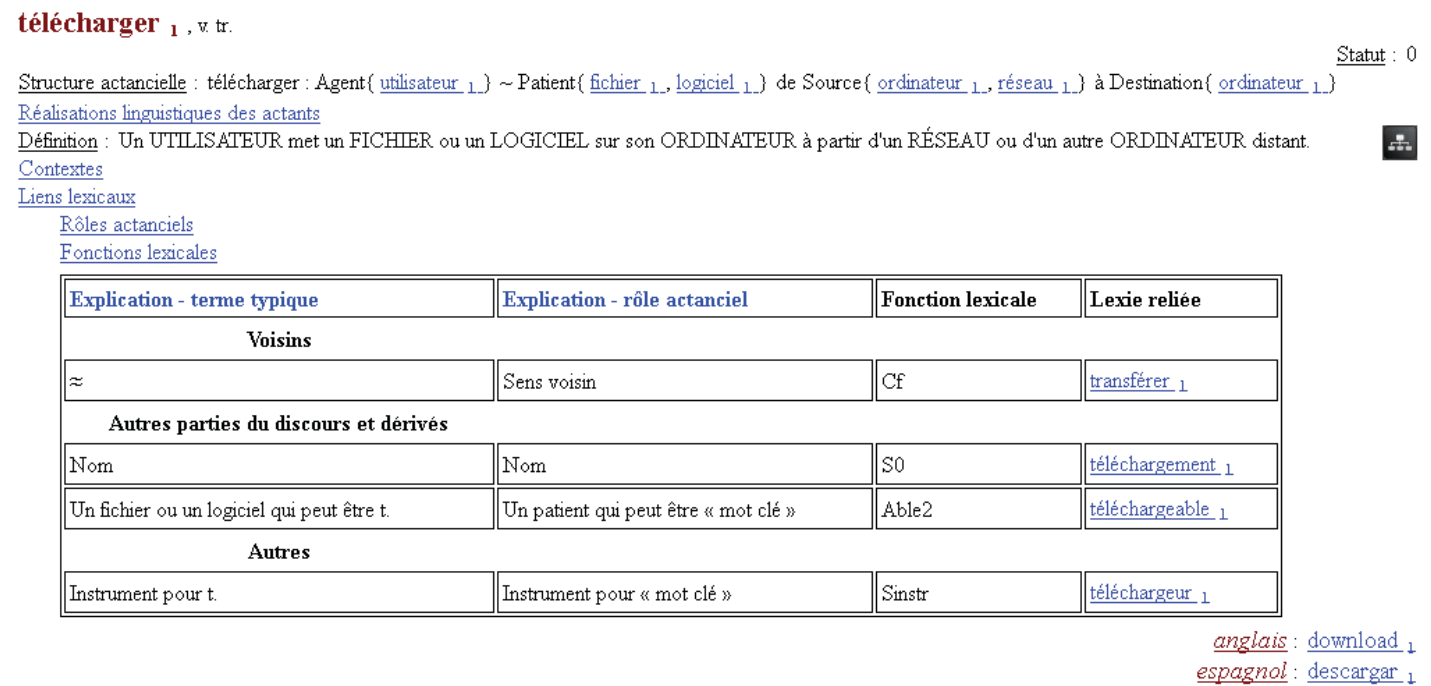

Figure 6 : Article télécharger dans le DiCoInfo

\section{Conclusion}

Quels enseignements pouvons-nous tirer des observations résumées dans les sections qui précèdent ? D'abord, peu importe la perspective adoptée, on s'entend sur le fait que le verbe doit être pris en compte d'une manière ou d'une autre dans l'analyse terminologique. Pour les uns (perspective conceptuelle), il exprime ce qu'exprime déjà un nom ou, encore, il rend compte d'une caractéristique d'un concept (étiqueté au moyen d'un nom); pour d'autres, il doit faire l'objet d'une description au même titre que le nom et occupe une place aussi importante que le terme de nature nominale (dans les optiques lexico-sémantiques); pour d'autres, enfin, les verbes ne participent pas tous au même degré à l'expression de connaissances spécialisées et figurent sur une sorte d'échelle (Lorente 2002; 2007).

Les intuitions que nous avons tenté de capter au moyen d'une petite enquête indiquent également que les locuteurs considèrent que les verbes peuvent avoir un sens spécialisé. Elles montrent en outre que certains verbes sont perçus comme étant plus spécialisés que d'autres ou, du moins, qu'on associe plus facilement des verbes à un domaine que d'autres. Les données de corpus ont montré que les verbes examinés ont une fréquence élevée et que parfois cette fréquence dépasse celle de la nominalisation correspondante.

On semble également s'entendre sur le fait que le verbe est au cœur d'une relation prédicat - argument où il joue le rôle de prédicat. Cette propriété apparaît en clair dans les travaux de sémantique lexicale et est représentée dans les modèles descriptifs que nous avons examinés. Elle apparaît également implicitement dans la perspective conceptuelle où le verbe peut être perçu comme l'élément connectant un concept et l'une de ses propriétés. La structure argumentale peut également servir de paramètre pour mieux comprendre la distribution des sens dans une ressource générale et une ressource spécialisée et peut guider l'établissement des équivalences. Le verbe est également au cœur de réseaux lexicaux identifiables au sein de domaines spécialisés et entretient avec d'autres unités lexicales (de nature nominale ou adjectivale) des liens plus ou moins étroits.

Enfin, les travaux convergent en ce sens qu'ils montrent que l'analyse du verbe peut mener à la découverte de caractéristiques conceptuelles ou lexicales propres aux domaines spécialisés. Le verbe joue un rôle 
important dans l'expression de faits spécialisés et, s'il est moins présent dans les réseaux conceptuels, il est au cœur de réseaux lexicaux.

\section{Remerciements}

J'aimerais remercier les étudiants qui ont accepté de répondre au questionnaire apparaissant dans le présent article. Je remercie également les collègues qui ont fourni des commentaires très constructifs sur l'ensemble de ce travail (en particulier Janine Pimentel qui a accepté de relire une version antérieure du présent texte). Différents organismes de financement ont soutenu ce travail : le Conseil de recherches en sciences humaines du Canada et le Fonds de recherche société et culture du Québec.

\section{Références}

Condamines, A. (1993). Un exemple d'utilisation de connaissances de sémantique lexicale : acquisition semiautomatique d'un vocabulaire de spécialité. Cahiers de lexicologie 62, pp. 25-65.

Fillmore, C. (1982). Frame Semantics. In Linguistics in the Morning Calm. Seoul: Hanshin Publishing Co., 111-137.

FrameNet (https://framenet.icsi.berkeley.edu/fndrupal/). Consulté le 27 février 2012.

Gross, G. (1994). Classes d'objets et description des verbes. Langages 115. 15-30.

L'Homme, M.-C. (1998). Le statut du verbe en langue de spécialité et sa description lexicographique. Cahiers de lexicologie 73(2), 61-84.

L'Homme, M.-C. (2003). Capturing the Lexical Structure in Special Subject Fields with Verbs and Verbal Derivatives: A Model for Specialized Lexicography. International Journal of Lexicography 16(4), 403-422.

L'Homme, M.C. (2009). Le DiCoInfo. Dictionnaire fondamental de l'informatique et de l'Internet. Montréal : Observatoire de linguistique Sens-Texte (OLST).

L'Homme, M.C. (2010). Designing Terminological Dictionaries for Learners based on Lexical Semantics: The representation of actants. In Fuertes-Olivera, P. (ed.). Specialised Dictionaries for Learners, Berlin/New York: De Gruyter, 141-153.

Lerat, P. (2002). Qu'est-ce que le verbe spécialisé ? Le cas du droit. Cahiers de Lexicologie 80, 201-211.

Lorente, M. (2002). Verbos y discurso especializado. Estudios de lingüística española (ELiEs) 16.

Lorente, M. (2007). Les unitats lèxiques verbals dels textos especialitzats. Redefinició d'una proposta de classificació. In Lorente, M. et al. (ed.) Estudis de lingüistics i de lingüistica aplicada en honor de M. Teresa Cabré Catellví. Volum II: De deixebles. Barcelona: Institut Universitari de Lingüística Aplicada de la Universitat Pompeu Fabra, Sèrie Monografies 11-12, 365-380.

Mel'čuk, I., A. Clas et A. Polguère (1995). Introduction à la lexicologie explicative et combinatoire, Louvain-la-Neuve : Duculot / Aupelf-UREF.

Pimentel, J. (2012, à paraître). Description de verbes juridiques au moyen de la sémantique des cadres ", Terminologie \& Ontologie: Théories et applications (Toth 2011), Annecy 2011, 26-27 mai 2011.

Pimentel, J. et L'Homme, M.C. (2011). Annotation syntaxico-sémantique de contextes spécialisés : application à la terminographie bilingue. .In van Campenhoudt, M., T. Lino et R. Costa (éd.). Passeurs de mots, passeurs d'espoir : lexicologie, terminologie et traduction face au défi de la diversité, Paris: Édition des archives contemporaines/Agence universitaire de la francophonie, pp. 651-670.

Pimentel, J. M.-C. L'Homme et M.E. Laneville (2012, à paraître). General and Specialized Lexical Resources: a Study on the Potential of Combining Efforts to Enrich Formal Lexicons. International Journal of Lexicography. 
Rey, A. (1979). La terminologie : noms et notions. Coll. « Que sais-je ? », Paris : Presses universitaires de France.

Robichaud, B. (2011). A Graph Visualization Tool for Terminology Discovery and Assessment. In Boguslavsky, I. et L. Wanner (eds.) Actes de la $5^{e}$ Conférence internationale sur la Théorie Sens-Texte (MTT'11), Barcelone, Espagne.

Ruppenhofer, J., M. Ellsworth, M. R. L. Petruck, C. Johnson and J. Scheffczyk (2010). FrameNet II: Extended theory and practice, http://framenet.icsi.berkeley.edu/. Consulté le 12 novembre 2011.

Sager, J.C. (1990). A Practical Course in Terminology Processing. Amsterdam/Philadelphia: John Benjamins.

Tellier, C. (2008). Verbes spécialisés en corpus médical : une méthode de description pour la rédaction d'articles terminographiques, Travail dirigé présenté au Département de linguistique et de traduction, Université de Montréal.

\footnotetext{
${ }^{1}$ Dans une ontologie de domaine on peut retrouver l'équivalent de formes verbales dans la formulation de propriétés (décrites sous forme de relations en OWL). Ainsi, une taxinomie contient généralement des classes d'entités (par exemple, Internet; Souris) et peut également renfermer des classes d'activité (Navigation; Clic). Les secondes sont reliées aux premières par une propriété du type aUsage (aUsage \{Internet, Navigation\}).

${ }^{2}$ Les données portent sur des formes lexicales désambiguïsées quant à la partie du discours, mais non quant au sens. C'est pourquoi nous avons mis de côté certaines formes paires verbe-nom.

${ }^{3}$ Ce critère peut paraître circulaire puisqu'il faut d'abord définir le statut terminologique des arguments. Toutefois, le statut terminologique des unités de nature nominale (et plus précisément des unités dénotant des entités) est beaucoup moins problématique. De plus, ce premier critère doit idéalement être combiné à d'autres (dérivation morphologique, relations paradigmatiques).

${ }^{4}$ Pour Lorente, le lien ne se limite pas uniquement à la parenté morphologique (bien que cette parenté soit envisagée). Ses critères permettent également d'accueillir des liens syntagmatiques.

${ }^{5}$ Accessible à l'adresse suivante : http://olst.ling.umontreal.ca/cgi-bin/dicoinfo/search.cgi.

${ }^{6}$ Accessible à l'adresse suivante : http://olst.ling.umontreal.ca/cgi-bin/dicoenviro/search_enviro.cgi
} 